\title{
Light scalar spectrum in extra-dimensional gauge theories
}

\author{
Enrico Rinaldi*t \\ SUPA and The Tait Institute, School of Physics and Astronomy, University of Edinburgh \\ Edinburgh, EH9 3JZ, UK \\ and \\ Kobayashi-Maskawa Institute, Nagoya University, \\ Nagoya, 464-8602, Japan \\ E-mail: e.rinaldiesms.ed.ac.uk
}

\section{Luigi Del Debbio}

SUPA and The Tait Institute, School of Physics and Astronomy, University of Edinburgh

Edinburgh EH9 3JZ, UK

E-mail: luigi.del.debbioded.ac.uk

\section{Alistair Hart}

EPCC, School of Physics and Astronomy, University of Edinburgh

Edinburgh EH9 3JZ, UK

E-mail: a.harteed.ac.uk

\begin{abstract}
The phase diagram of five-dimensional SU(2) gauge theories with one compactified dimension on anisotropic lattices has a rich structure. In this contribution we show how to control non-perturbatively the scale hierarchy between the cut-off and the compactification scale in the bare parameter space. There exists a set of strong bare couplings where the the five-dimensional lattice theory can be described by an effective four-dimensional theory with a scalar field in the adjoint representation.

We present a detailed study of the light scalar spectrum as it arises from the non-perturbative dynamics of the full five-dimensional lattice theory. We also investigate the mixing with scalar glueball states in the attempt to further establish the extra-dimensional nature of light scalar states.
\end{abstract}

The 30 International Symposium on Lattice Field Theory - Lattice 2012,

June 24-29, 2012

Cairns, Australia

\footnotetext{
* Speaker.

${ }^{\dagger}$ The author is supported by a SUPA prize studenship and a JSPS short-term fellowship.
} 


\section{Introduction}

An elementary scalar field in any renormalisable four-dimensional quantum field theory will get an additive mass renormalization proportional to the second power of the ultra-violet (UV) cutoff. It is a well known problem that is usually referred to as the naturalness problem regarding the elementary scalar Higgs field in the Standard Model.

Theoretical efforts have been made in order to mitigate or completely avoid the quadratic cut-off dependence of the elementary scalar mass. It is interesting to note that some classes of extradimensional models can resolve this issue in a simple way. This is due to the presence of an extended gauge symmetry protecting the scalar mass from quantum correction, because in these models the scalar field is actually one of the components of the higher-dimensional gauge field.

Let us consider, for sake of definiteness, a $\mathrm{SU}\left(N_{c}\right)$ Yang-Mills theory in a five-dimensional Euclidean space-time defined by the following action:

$$
\mathscr{S}_{E}^{5 d}=\int d^{5} \times \frac{1}{2 g_{5}^{2}} \operatorname{Tr} F_{M N}^{2}
$$

where the field-strength tensor $F_{M N}$ is defined generalising the four-dimensional case with $M, N=$ $\{1, \ldots, 5\}$.

Due to its naive non-renormalizability, a UV cut-off $\Lambda_{\mathrm{UV}}$ is needed and the regulated theory can be consider as an effective physics description at energies $E \ll \Lambda_{\mathrm{UV}}$ (where the details of the regularization can be neglected). This full five-dimensional theory can be dimensionally reduced by compactifying the extra dimension on a small circle $S^{1}$ of radius $R$. Below the compactification scale $\Lambda_{\mathrm{R}} \sim R^{-1}$, the physics is effectively described by a four-dimensional gauge theory with an additional scalar degree of freedom coming from the compactified fifth component of the higherdimensional gauge field. This low-energy effective action can be written as

$$
\mathscr{S}_{\text {eff }}^{4 d} \sim \int d^{4} x\left[\frac{1}{2} \operatorname{Tr}\left(F_{\mu v} F^{\mu v}\right)+\operatorname{Tr}\left(D_{\mu} A_{5}\right)^{2}\right],
$$

where $A_{5}$ is a scalar field in the adjoint representation of the gauge group. The extra-dimensional origin of $A_{5}$ prevents its tree-level mass to be different from zero. However, one can calculate radiative corrections to the scalar mass within the validity regime $\Lambda_{R} \ll \Lambda_{U V}$ of this four-dimensional (renormalisable) theory using perturbation theory. Different approaches, at the one and two-loop level [1,2], proved the finiteness of the scalar mass and its independence of the UV cut-off. This perturbative result is further confirmed in Ref. [3] where the authors used the full five-dimensional theory (see Eq. (1.1)) with two explicit UV completions (string theory and lattice field theory) in order to properly regulate the high-energy contributions. Although the regulating schemes are very different from each other, the radiative corrections to the scalar mass of the low-energy fourdimensional theory agree with each other when the compactification scale is well separated from the scale where the details of the regularization appear. The renormalized scalar mass $m_{5}$ is

$$
m_{5}^{2}=\frac{9 g_{5}^{2} N_{c}}{32 \pi^{3} R^{3}} \zeta(3)
$$

where $\zeta$ is the Riemann Zeta-function. This is a very interesting results because it seems to be universal for any UV completion of the five-dimensional theory which respects locality and gaugeinvariance. 
It is then natural to ask what is the validity of these predictions when the coupling constant is not small and perturbation theory is not reliable. In particular, already in four dimensions, YangMills theories develop a mass gap non-perturbatively and confine at low-energy, a feature that is completely missed by perturbation theory. By studying the non-perturbative low-energy dynamics of the theory described by Eq. (1.1), we aim at understanding the fate of Eq. (1.3). In particular it is important to know whether the independence of $\Lambda_{\mathrm{UV}}$ and the functional dependence on $\Lambda_{\mathrm{R}}$ are preserved in the strong-coupling regime.

The results of our study are also presented in Ref. [4] and in the following we summarise and underline the main original contributions.

\section{The lattice model}

Non-perturbative low-energy physics of a non-renormalizable $\mathrm{SU}\left(N_{c}\right)$ Yang-Mills theory can be studied using lattice Monte Carlo simulations. The lattice acts as a gauge-invariant regulator at the UV level and long-distance observables can be studied numerically. The caveat is that systematic and statistical errors are introduced and great care should be taken in order to deal with them. Our lattice discretization of the action in Eq. (1.1) is an anisotropic Wilson plaquette action with two independent coupling constants:

$$
\mathscr{S}_{W}=\beta_{4} \sum_{x ; 1 \leq \mu \leq \nu \leq 4}\left[1-\frac{1}{2} \operatorname{Re} \operatorname{Tr} P_{\mu \nu}(x)\right]+\beta_{5} \sum_{x ; 1 \leq \mu \leq 4}\left[1-\frac{1}{2} \operatorname{Re} \operatorname{Tr} P_{\mu 5}(x)\right] .
$$

The four-dimensional plaquette $P_{\mu \nu}$ and the extra-dimensional one $P_{\mu 5}$ have different couplings $\beta_{4}$ and $\beta_{5}$ allowing for two different lattice spacings to be varied independently: $a_{4}$ in the fourdimensional sub-lattice and $a_{5}$ along the extra dimension. The physical lengths of the lattice are determined by the following quantities: $L_{4}=a_{4} N_{4}$ and $L_{5}=2 \pi R=a_{5} N_{5}$; the three-dimensional volume will be $V=\left(L_{4}\right)^{3}$, with temporal extent $L_{T}=L_{4}$ and extra-dimensional size $L_{5}$. The setup just described is well suited to study a physical system with a compact extra dimension, because of the freedom of adjusting both $a_{5}$ and $N_{5}$ independently of $a_{4}$ and $V$ in order to obtain any possible scale separation between $\Lambda_{\mathrm{R}}$ and $\Lambda_{\mathrm{UV}}$.

Previous studies in the literature have explored the phase diagram of this lattice model $[5,6,7]$ and a summary of the most recent results can be found in Ref. [4]. We focus on the region of parameter space $\left(\beta_{4}, \beta_{5}, N_{4}, N_{5}\right)$ with $N_{5}<N_{4}$ and $\beta_{4}<\beta_{5}$ where a dimensionally reduced phase appears thanks to the compactification of the extra dimension. In this phase, the long-distance physics of the system is expected to be described by the four-dimensional effective theory of Eq. (1.2) with a light scalar particle whose mass is perturbatively given by Eq. (1.3).

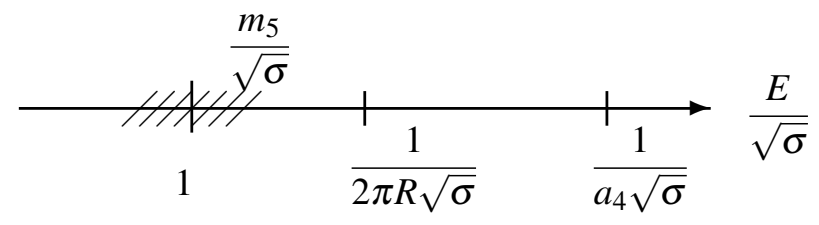

Figure 1: The figure shows the desired separation of energy scales. The scales are all expressed in terms of the four-dimensional string tension that characterises the low-energy physics of the theory. 


\section{Long-distance physics and scalar spectrum}

The perturbative result of Eq. (1.3) holds when $\Lambda_{\mathrm{R}} \ll \Lambda_{\mathrm{UV}}$ and the details of the regularization can be neglected. Also, the low-energy physics, whose energy scale is determined by the string tension $\sqrt{\sigma}$, should be separated from both the compactification and the cut-off scale in order for our results to be meaningful. Therefore, the first step of our study is to determine non-perturbatively the energy scales of the physical system. The desired hierarchy is represented schematically in Fig. 2, where the string tension is used to set the scale. The simulation's strategy is straightforward in principle: by fixing a point in the bare lattice parameter space, we are choosing a given separation of scales. The energy scales of the system are determined by measuring physical observables via numerical Monte Carlo simulations.

We measure $a_{4} \sqrt{\sigma}$ and $a_{4} m_{5}$ by studying the large-time exponential decay of correlators of suitable lattice operators. The string tension is extracted from the ground state mass of correlators of long spatial Polyakov loops. On the other hand, the scalar mass requires us to construct operators that transform as scalars in three dimensions and with positive parity and charge (belonging to the $0^{++}$irreducible representation in standard spectroscopic notation). We distinguish between two different kind of operators in the scalar channel: those constructed using links $U_{5}(x)$ in the compact fifth direction (generically called scalar operators in the following) and those built using only spatial links $U_{i}(x)$ (called glueball operators). For the first kind, we use compact Polyakov loop operators, that is gauge-invariant combinations of Polyakov loops winding around the extra fifth dimension. In particular, we choose two different combinations

$$
\mathscr{O}_{1}(t)=\sum_{x} \operatorname{Tr}\left[l_{5}(x, t)\right] ; \quad l_{5}(x, t)=\prod_{j=1}^{N_{5}} U_{5}\left(x+j a_{5} \hat{5}, t\right),
$$

and

$$
\mathscr{O}_{2}(t)=\sum_{x} \operatorname{Tr}\left[\phi(x, t) \phi^{\dagger}(x, t)\right] ; \quad \phi(x, t)=\frac{l_{5}-l_{5}^{\dagger}}{2} .
$$

We average the correlators over the extra-dimensional coordinate and correlate along the temporal coordinate.

For the operator in Eq. (3.2) we apply the smearing procedure introduced in Ref. [8] for a scalar Higgs field: the operator $\phi$ is replaced by a smeared version that consists of a gauge-invariant combination of parallel transporters in the three-dimensional spatial subspace. Operators $\mathscr{O}_{1}$ and $\mathscr{O}_{2}$ are expected to couple mainly with scalar states of extra-dimensional nature. To create oper-

a)

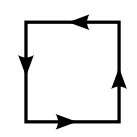

b)

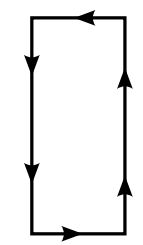

c)

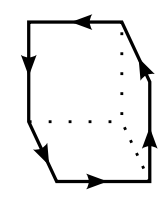

Figure 2: Wilson loops used in the construction of glueball operators in the scalar channel. Each of these three operators is smeared in order to construct a larger variational ansatz.

ators coupling to scalar glueball states, instead, we use symmetrized combinations of the spatial 
Wilson loops in Fig. 3. These operators project only onto the scalar representation of the threedimensional cubic symmetry group are then cross-correlated together with operators $\mathscr{O}_{1}$ and $\mathscr{O}_{2}$; we refer to these scalar glueball operators as $\mathscr{O}_{a}, \mathscr{O}_{b}$ and $\mathscr{O}_{c}$ built starting respectively from the path a), b) and c) in Fig. 3. The glueball operators are smeared according to the improved blocking algorithm described in Ref. [9].

The inclusion of glueball operators in the study of the light scalar spectrum of this five-dimensional lattice model is necessary in order to better understand the fate of Eq. (1.3). The presence of light scalar glueballs could induce mixing with the scalar state we expect perturbatively due to the compactification mechanism described in Sec. 1. Thanks to our extended set of operators, we can use a variational ansatz to study the scalar spectrum and extract informations about the mixing of the ground state with glueball operators. The variational procedure finds the linear combination of the available operators $\left(\mathscr{O}_{1}, \ldots, \mathscr{O}_{c}\right)$ that best approximates the wavefunction of the lowest mass eigenstate. The relative contribution of each operator to the mass eigenstate can be used to infer the nature of the state: if the main contribution is due to glueball operators, the mass extracted is likely to be associated to a glueball state. On the other hand, a dominant contribution coming from the scalar operators would confirm the extra-dimensional nature of the extracted mass. This study can be performed on the lightest excitations of the spectrum as well.

Due to the high computational cost of such a measurement, we employed the full variational method on a subset of the phase diagram explored. We choose a lattice of size $L_{4}=10 a_{4}$ and $L_{5}=$ $4 a_{5}$, but with an extended temporal direction $L_{T}=2 L_{4}$ that helped us reduce finite-size systematic effects. Then we fix a line in the parameter space corresponding to a fixed ratio of the coupling constants $\gamma=\sqrt{\beta_{4} / \beta_{5}} \approx 1.54$ and we simulate different points with $\beta=\sqrt{\beta_{4} \beta_{5}} \in\{1.72, \ldots, 1.77\}$. These points correspond to a fixed separation between the compactification and the cut-off scale $\Lambda_{\mathrm{UV}} / \Lambda_{\mathrm{R}} \approx 2$ and increasing $\beta$ values leads to smaller extra-dimensional radii. The perturbative expectation in this region is that the scalar mass will increase with $\beta$.
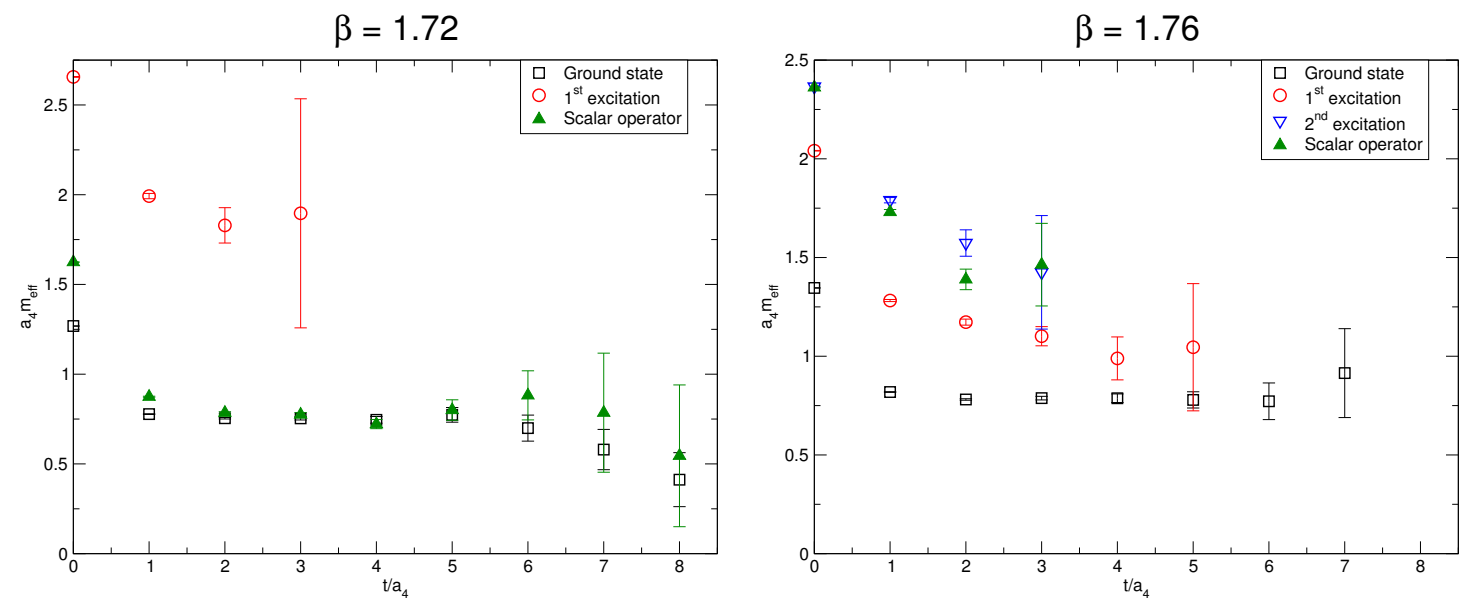

Figure 3: Example of effective mass plateaux for two different values of $\beta$ at fixed $\gamma=1.5433$. (left) At $\beta=1.72$ the mass of the low-lying scalar state obtained from the variational ansatz is compatible with the one we measured using only the scalar operator $\mathscr{O}_{2}$. (right) At $\beta=1.76$ the scalar operator $\mathscr{O}_{2}$ yields a mass which is compatible with the second excitations of the scalar spectrum. 
In Fig. 3 we show the effective masses extracted from the variational analysis of the scalar correlators for two different $\beta$ values. At the smallest $\beta$, the variational ground state is the same that we obtain using scalar operators of type $\mathscr{O}_{2}$ and this is a first indication that the lowest propagating state would come from the dimensional reduction of the full five-dimensional gauge theory. On the contrary, at the largest $\beta$, the correlators of scalar operators gives an effective mass which is definitively heavier than the one of the variational ground state. The scalar ground state at $\beta=1.76$ seems to be dominated by the contributions of glueball operators. This can be verified by looking at the relative projections of each operator onto the ground state; the results are summarised in Fig. 3 for all the $\beta$ values included in our study. When $\beta$ is increased at fixed $\gamma$, the contribution to the ground state is first dominated by scalar operators, but glueball operators start to mix and then become dominant at large $\beta$. The emerging physical picture is that the effective theory describing the long-distance physics in this region of the bare coupling space, becomes more and more four-dimensional with the lowest scalar spectrum dominated by non-perturbative gauge excitations (glueballs). Any sign of the extra-dimensional UV nature of the system is lost when the excitations of the scalar field coming from the compactification mechanism become heavy and decouple from the low-energy dynamics. This picture is in agreement with earlier results [6] that probed the fourdimensional nature of the effective theory by carefully studying the string tension dependence on the lattice couplings.

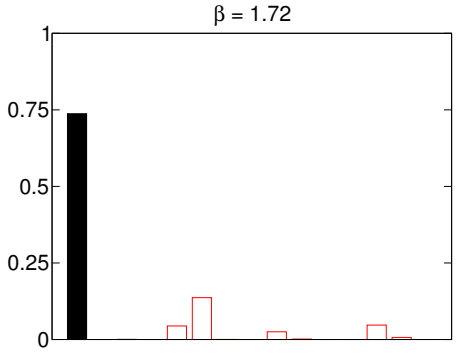

$\beta=1.75$

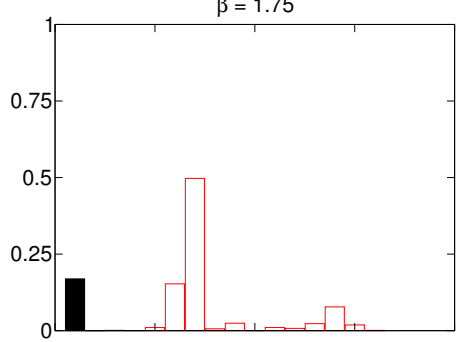

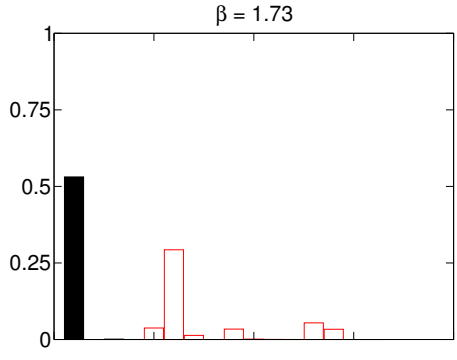

$\beta=1.76$

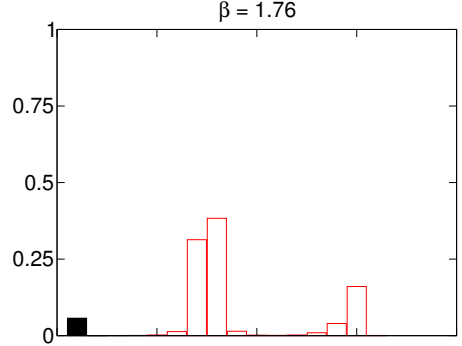

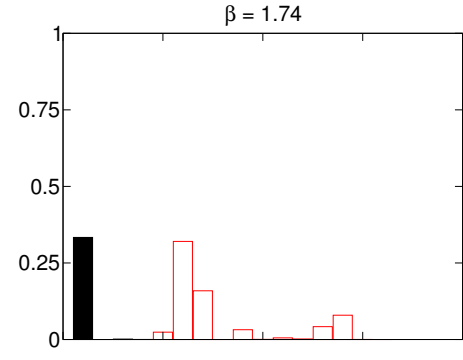

$\beta=1.77$

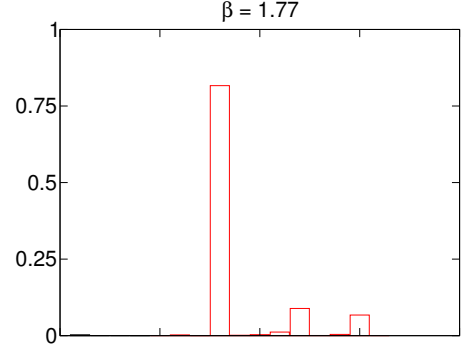

Figure 4: Relative projection of the ground state onto each of the operators in the variational set for simulations at fixed $\gamma=1.5433$ and increasing $\beta$. Filled symbols correspond to the set of smeared scalar operators, whereas the open symbols refer to the smeared versions of glueball operators.

\section{Conclusions}

We found a region of the bare parameters of a five-dimensional lattice model where dimensional reduction through compactification of the extra dimension takes place. In this region we studied the light spectrum in the scalar channel and we determined non-perturbatively the physical energy scales $\Lambda_{R}$ and $\Lambda_{U V}$. When the desired separation of scales is found (see Fig. 2), we expect 
the low-energy dynamics to be described by an effective four-dimensional gauge theory coupled to an additional scalar field. This effective description is confirmed by our measurements of the non-perturbative spectrum. For a specific region of the parameter space, we have shown that the lightest state propagating in the scalar channel has a negligible mixing with glueball states and its mass can be unambiguously identified with $m_{5}$. Moreover, the functional dependence of this nonperturbative scalar mass is found to be consistent with the perturbative prediction of Eq. (1.3). The results of this study and of the one in Ref. [6] can be considered as a good starting point for investigating the realisation of the compactification mechanism from five to four dimensions at the non-perturbative level.

\section{References}

[1] Y. Hosotani, Dynamical mass generation by compact extra dimensions, Phys. Lett., B126 (1983) 309; Y. Hosotani, N. Maru, K. Takenaga, T. Yamashita, Two loop finiteness of Higgs mass and potential in the gauge-Higgs unification, Prog. Theor. Phys., 118 (2007) 1053-1068.

[2] H. Cheng, K. Matchev, M. Schmaltz, Radiative corrections to Kaluza-Klein masses, Phys. Rev., D66, (2002) 036005 .

[3] L. Del Debbio, E. Kerrane, R. Russo, Mass corrections in string theory and lattice field theory, Phys. Rev., D80, (2009) 025003.

[4] L. Del Debbio, A. Hart, E. Rinaldi, Light scalars in strongly-coupled extra-dimensional theories, JHEP, 07 (2012) 178

[5] S. Ejiri, J. Kubo, M. Murata, A study on the nonperturbative existence of Yang-Mills theories with large extra dimensions, Phys. Rev., D62 (2000) 105025;

S. Ejiri, S. Fujimoto, J. Kubo, Scaling laws and effective dimension in lattice SU(2) Yang-Mills theory with a compactified extra dimension, Phys.Rev., D66, (2002) 036002.

[6] P. de Forcrand, A. Kurkela, M. Panero, The phase diagram of Yang-Mills theory with a compact extra dimension, JHEP, 06 (2010) 050.

[7] F. Knechtli, M. Luz, A. Rago, On the phase structure of five-dimensional SU(2) gauge theories with anisotropic couplings, Nucl.Phys., B856 (2012) 74-94.

[8] N. Irges, F. Knechtli, Lattice Gauge Theory Approach to Spontaneous Symmetry Breaking from an Extra Dimension, Nucl. Phys., B775 (2007) 283-311.

[9] B. Lucini, A. Rago, E. Rinaldi, Glueball masses in the large N limit, JHEP, 08 (2010) 119. 